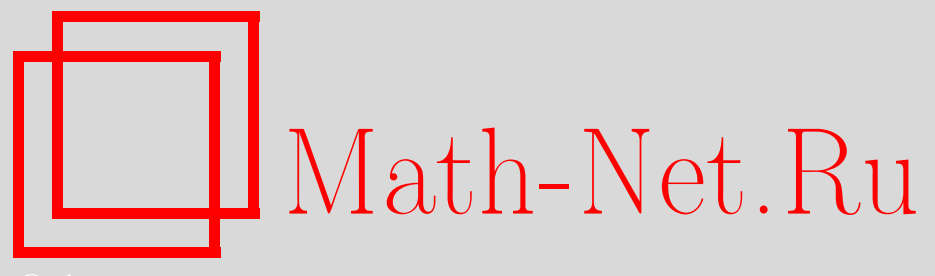

В. А. Емеличев, К. Г. Кузьмин, Анализ устойчивости строго эффективного решения одной векторной задачи булева программирования в метрике $l_{1}$, Дискрет. матем., 2004, том 16, выпуск 4, 14-19

DOI: https://doi.org/10.4213/dm171

Использование Общероссийского математического портала Math-Net.Ru подразумевает, что вы прочитали и согласны с пользовательским соглашением http://www . mathnet.ru/rus/agreement

Параметры загрузки:

IP : 54.157 .27 .8

26 апреля 2023 г., 13:39:22 
УдК 519.10

\title{
Анализ устойчивости строго эффективного решения одной векторной задачи булева программирования в метрике $l_{1}$
}

\author{
() 2004 г. В. А. Емеличев, К. Г. Кузьмин
}

\begin{abstract}
Рассматривается векторная (многокритериальная) задача булева программирования с частными критериями, являющимися проекциями линейных функций на $\mathbf{R}_{+}$. Указана граница изменений коэффициентов таких функций в метрике $l_{1}$, сохраняющих строгую эффективность решения.

Работа выполнена при поддержке Государственной программы фундаментальных исследований Республики Беларусь «Математические структуры» 29.
\end{abstract}

Исходная информация большинства прикладных задач дискретной оптимизации носит приближенный характер, она во многих случаях неточна и некорректна. В связи с этим актуальным является анализ устойчивости решений при изменениях параметров задачи. Исследованию различных аспектов устойчивости скалярных и векторных задач дискретной оптимизации посвящен ряд публикаций (см., например, монографию [1] и обзоры [2-5]). В данной статье продолжаются начатые в [6-10] исследования радиусов устойчивости решений векторных задач дискретной оптимизации с различными видами частных критериев и разнообразными принципами оптимальности (функциями выбора). Здесь выведена формула радиуса устойчивости оптимального по Смейлу (строго эффективного) решения векторной задачи минимизации невязок системы линейных булевых неравенств, то есть задачи минимизации проекций линейных булевых функций на $\mathbf{R}_{+}$.

Пусть $m \geqslant 1, n \geqslant 2, A_{i}-i$-я строка матрицы $A=\left[a_{i j}\right]_{m \times n} \in \mathbf{R}^{m n}, b \in \mathbf{R}^{m}$, $X \subseteq \mathbf{E}^{n}=\{0,1\}^{n},|X| \geqslant 2$. Введем в рассмотрение операцию проектирования вектора $a=\left(a_{1}, a_{2}, \ldots, a_{m}\right) \in \mathbf{R}^{m}$ на неотрицательный ортант $\mathbf{R}_{+}^{m}$, результатом которой является вектор

$$
a^{+}=\left(a_{1}^{+}, a_{2}^{+}, \ldots, a_{m}^{+}\right)
$$

где $a_{i}^{+}=\left[a_{i}\right]^{+}=\max \left\{0, a_{i}\right\}, i \in N_{m}=\{1,2, \ldots, m\}$.

На множестве решений $X$ зададим векторный критерий

$$
[A x+b]^{+} \rightarrow \min _{x \in X}
$$

частными критериями которого являются проекции $\left[A_{i} x+b_{i}\right]^{+}, i \in N_{m}$, линейных функций. Легко видеть, что минимизация этих проекций на множестве $X$ равносильна минимизации невязок (уклонений) следующей системы линейных булевых неравенств:

$$
A_{i} x+b_{i} \leqslant 0, \quad i \in N_{m}, \quad x \in X .
$$


Отметим также, что операции проектирования на простейшие выпуклые множества, такие, как неотрицательный ортант, гиперплоскость, полупространство, линейное многообразие и другие, лежат в основе различных вариантов фейеровских итерационных процессов, применяемых для численного анализа собственных и несобственных задач линейного программирования $[11,12]$.

Введем традиционное множество Смейла $S^{m}(A, b)$, то есть множество строго эффективных решений $[13,14] m$-критериальной задачи (1):

$$
S^{m}(A, b)=\left\{x \in X: S^{m}(x, A, b)=\varnothing\right\},
$$

где

$$
\begin{aligned}
S^{m}(x, A, b) & =\left\{x^{\prime} \in X \backslash\{x\}: g\left(x, x^{\prime}, A, b\right) \leqslant 0_{(m)}\right\}, \\
g\left(x, x^{\prime}, A, b\right) & =\left(g_{1}\left(x, x^{\prime}, A_{1}, b_{1}\right), g_{2}\left(x, x^{\prime}, A_{2}, b_{2}\right), \ldots, g_{m}\left(x, x^{\prime}, A_{m}, b_{m}\right)\right), \\
g_{i}\left(x, x^{\prime}, A_{i}, b_{i}\right) & =\left[A_{i} x^{\prime}+b_{i}\right]^{+}-\left[A_{i} x+b_{i}\right]^{+}, \quad i \in N_{m}, \\
0_{(m)} & =(0,0, \ldots, 0) \in \mathbf{R}^{m} .
\end{aligned}
$$

Очевидно, что любое такое решение является эффективным, то есть оптимальным по Парето. Отметим также, что строгую эффективность решения можно трактовать как векторный аналог единственности оптимального решения скалярной (однокритериальной) задачи, поскольку в этом случае $\left|S^{1}(A, b)\right|=1$.

Для всякого натурального числа $q$ в $q$-мерном действительном пространстве $\mathbf{R}^{q}$ зададим метрику $l_{1}$, то есть под нормой вектора $z=\left(z_{1}, z_{2}, \ldots, z_{q}\right)$ будем понимать число

$$
\|z\|=\sum_{i \in N_{q}}\left|z_{i}\right|
$$

a под нормой $\|A\|$ матрицы $A=\left[a_{i j}\right]_{m \times n} \in \mathbf{R}^{m n}$ будем понимать норму вектора $\left(a_{11}, a_{12}, \ldots, a_{m n-1}, a_{m n}\right) \in \mathbf{R}^{m n}$.

Следуя $[6,7,9,10]$, радиусом устойчивости решения $x \in S^{m}(A, b)$ назовем число

$$
\rho^{m}(x, A, b)= \begin{cases}\sup \Xi, & \text { если } \Xi \neq \varnothing, \\ 0, & \text { если } \Xi=\varnothing,\end{cases}
$$

где

$$
\Xi=\left\{\varepsilon>0: \forall\left(A^{\prime}, b^{\prime}\right) \in \Omega(\varepsilon) \quad\left(x \in S^{m}\left(A+A^{\prime}, b+b^{\prime}\right)\right)\right\},
$$

$\Omega(\varepsilon)$ - множество таких пар $\left(A^{\prime}, b^{\prime}\right)$, что $A^{\prime} \in \mathbf{R}^{m n}, b^{\prime} \in \mathbf{R}^{m},\left\|A^{\prime}\right\|+\left\|b^{\prime}\right\|<\varepsilon$. Таким образом, радиус устойчивости задает предельный уровень возмущений параметров частных критериев в пространстве $\mathbf{R}^{m n+m}$ с метрикой $l_{1}$, при которых сохраняется строгая эффективность решения $x$. Ранее исследования устойчивости в пространстве параметров с такой метрикой проводились для скалярных комбинаторных задач в $[15,16]$.

Для вывода формулы радиуса устойчивости нам понадобится ряд вспомогательных утверждений.

Учитывая очевидные соотношения

$$
c^{+}=\frac{c+|c|}{2} \leqslant|c|, \quad|c|-|d| \leqslant|c+d| \leqslant|c|+|d|,
$$

справедливые для любых чисел $c, d \in \mathbf{R}$, легко доказать неравенства

$$
c^{+}-[-d]^{+} \leqslant[c+d]^{+} \leqslant c^{+}+d^{+} \leqslant|c|+|d| .
$$


Отсюда после несложных преобразований для любого индекса $i \in N_{m}$ и всякого решения $x \in X$ находим, что

$$
\max \left\{\left[A_{i} x+b_{i}\right]^{+},\left[-\left(A_{i} x+b_{i}\right)\right]^{+}\right\} \leqslant\left\|A_{i}\right\|+\left|b_{i}\right| .
$$

Кроме того, справедливо следующее более сильное утверждение.

Свойство 1. Для любых $x, x^{\prime} \in X u i \in N_{m}$ имеет место неравенство

$$
\left[A_{i} x+b_{i}\right]^{+}+\left[-\left(A_{i} x^{\prime}+b_{i}\right)\right]^{+} \leqslant\left\|A_{i}\right\|+\left|b_{i}\right| \text {. }
$$

Действительно, если $A_{i} x+b_{i}>0$ и $A_{i} x^{\prime}+b_{i}<0$, то справедливы соотношения

$$
\left[A_{i} x+b_{i}\right]^{+}+\left[-\left(A_{i} x^{\prime}+b_{i}\right)\right]^{+}=A_{i} x-A_{i} x^{\prime} \leqslant\left\|A_{i}\right\| \leqslant\left\|A_{i}\right\|+\left|b_{i}\right| .
$$

Во всех остальных случаях хотя бы одна из величин $\left[A_{i} x+b_{i}\right]^{+}$или $\left[-\left(A_{i} x^{\prime}+b_{i}\right)\right]^{+}$ равна нулю, и поэтому в силу (3) имеет место неравенство (4).

Свойство 2. Для любых $x, x^{\prime} \in X u i \in N_{m}$ справедливо неравенство

$$
g_{i}\left(x, x^{\prime}, A_{i}+A_{i}^{\prime}, b_{i}+b_{i}^{\prime}\right) \geqslant g_{i}\left(x, x^{\prime}, A_{i}, b_{i}\right)-\left\|A_{i}^{\prime}\right\|-\left|b_{i}^{\prime}\right| .
$$

Действительно, используя неравенства (2) и свойство 1, получаем, что

$$
\begin{aligned}
g_{i}\left(x, x^{\prime}, A_{i}+A_{i}^{\prime}, b_{i}+b_{i}^{\prime}\right) & =\left[A_{i} x^{\prime}+A_{i}^{\prime} x^{\prime}+b_{i}+b_{i}^{\prime}\right]^{+}-\left[A_{i} x+A_{i}^{\prime} x+b_{i}+b_{i}^{\prime}\right]^{+} \\
& \geqslant\left[A_{i} x^{\prime}+b_{i}\right]^{+}-\left[-\left(A_{i}^{\prime} x^{\prime}+b_{i}^{\prime}\right)\right]^{+}-\left[A_{i} x+b_{i}\right]^{+}-\left[A_{i} x^{\prime}+b_{i}^{\prime}\right]^{+} \\
& =g_{i}\left(x, x^{\prime}, A_{i}, b_{i}\right)-\left[A_{i} x^{\prime}+b_{i}^{\prime}\right]^{+}-\left[-\left(A_{i}^{\prime} x^{\prime}+b_{i}^{\prime}\right)\right]^{+} \\
& \geqslant g_{i}\left(x, x^{\prime}, A_{i}, b_{i}\right)-\left\|A_{i}^{\prime}\right\|-\left|b_{i}^{\prime}\right| .
\end{aligned}
$$

Лемма 1. Если для некоторого индекса $i \in N_{m}$

$$
g_{i}\left(x, x^{\prime}, A_{i}+A_{i}^{\prime}, b_{i}+b_{i}^{\prime}\right) \leqslant 0,
$$

то справедливо неравенство

$$
g_{i}^{+}\left(x, x^{\prime}, A_{i}, b_{i}\right) \leqslant\left\|A_{i}^{\prime}\right\|+\left|b_{i}^{\prime}\right| .
$$

Действительно, если $g_{i}\left(x, x^{\prime}, A_{i}, b_{i}\right) \leqslant 0$, то неравенство (6) очевидно, а если $g_{i}\left(x, x^{\prime}, A_{i}, b_{i}\right)>0$, то, используя свойство 2 и неравенство (5), находим, что

$g_{i}^{+}\left(x, x^{\prime}, A_{i}, b_{i}\right)=g_{i}\left(x, x^{\prime}, A_{i}, b_{i}\right) \leqslant g_{i}\left(x, x^{\prime}, A_{i}+A_{i}^{\prime}, b_{i}+b_{i}^{\prime}\right)+\left\|A_{i}^{\prime}\right\|+\left|b_{i}^{\prime}\right| \leqslant\left\|A_{i}^{\prime}\right\|+\left|b_{i}^{\prime}\right|$.

Введем обозначение

$$
\alpha\left(x, x^{\prime}, A, b\right)=\sum_{i \in N_{m}} g_{i}^{+}\left(x, x^{\prime}, A_{i}, b_{i}\right) .
$$

Лемма 2. Если $\alpha\left(x, x^{\prime}, A, b\right)>0$, то для всякого числа $\varphi$, удовлетворяющего неравенствам

$$
0<\varphi \leqslant \alpha\left(x, x^{\prime}, A, b\right)
$$

справедлива формула

$$
\forall\left(A^{\prime}, b^{\prime}\right) \in \Omega(\varphi) \quad \exists i \in N_{m} \quad\left(g_{i}\left(x, x^{\prime}, A_{i}+A_{i}^{\prime}, b_{i}+b_{i}^{\prime}\right)>0\right) .
$$


Доказательство. Предположим, что, напротив, существует такая возмущающая пара $\left(A^{\prime}, b^{\prime}\right) \in \Omega(\varphi)$, что для любого индекса $i \in N_{m}$ справедливо неравенство

$$
g_{i}\left(x, x^{\prime}, A_{i}+A_{i}^{\prime}, b_{i}+b_{i}^{\prime}\right) \leqslant 0 .
$$

Тогда согласно лемме 1 для всякого индекса $i \in N_{m}$ имеет место неравенство (6). Поэтому с учетом включения $\left(A^{\prime}, b^{\prime}\right) \in \Omega(\varphi)$ верны соотношения

$$
\alpha\left(x, x^{\prime}, A, b\right)=\sum_{i \in N_{m}} g_{i}^{+}\left(x, x^{\prime}, A_{i}, b_{i}\right) \leqslant \sum_{i \in N_{m}}\left(\left\|A_{i}^{\prime}\right\|+\left|b_{i}^{\prime}\right|\right)=\left\|A^{\prime}\right\|+\left\|b^{\prime}\right\|<\varphi,
$$

что противоречит условию (7).

Лемма 2 доказана.

Лемма 3. Пусть $x \in S^{m}(A, b), x^{\prime} \in X \backslash\{x\}$. Тогда для любых чисел

$$
\xi_{i}>g_{i}^{+}\left(x, x^{\prime}, A_{i}, b_{i}\right), \quad i \in N_{m},
$$

cуществует пара $\left(A^{\prime}, b^{\prime}\right) \in \Omega(\varepsilon)$, где $\varepsilon>\xi=\sum_{i \in N_{m}} \xi_{i}$, такая, что $x \notin S^{m}\left(A+A^{\prime}, b+b^{\prime}\right)$. Доказательство. Прежде всего отметим, что все числа $\xi_{i}, i \in N_{m}$, удовлетворяющие неравенствам (9), положительны.

Далее, введем обозначение

$$
N(x)=\left\{j \in N_{n}: x_{j}=1\right\}
$$

и рассмотрим два возможных случая.

В первом случае $N\left(x^{\prime}\right) \backslash N(x) \neq \varnothing$. Положим $b^{\prime}=0_{(m)}$ и, зафиксировав произвольный индекс $q \in N\left(x^{\prime}\right) \backslash N(x)$, зададим элементы матрицы $A^{\prime}=\left[a_{i j}^{\prime}\right]_{m \times n}$ формулой

$$
a_{i j}^{\prime}= \begin{cases}-\xi_{i}, & \text { если } i \in N_{m}, j=q, \\ 0, & \text { если } i \in N_{m}, j \neq q .\end{cases}
$$

Тогда с учетом неравенств (9) для любого индекса $i \in N_{m}$ справедливы соотношения

$$
\begin{aligned}
g_{i}\left(x, x^{\prime}, A_{i}+A_{i}^{\prime}, b_{i}+b_{i}^{\prime}\right) & =\left[A_{i} x^{\prime}+b_{i}-\xi_{i}\right]^{+}-\left[A_{i} x+b_{i}\right]^{+} \\
& \leqslant\left[\left[A_{i} x^{\prime}+b_{i}\right]^{+}-\xi_{i}\right]^{+}-\left[A_{i} x+b_{i}\right]^{+} \\
& \leqslant\left[\left[A_{i} x^{\prime}+b_{i}\right]^{+}-g_{i}^{+}\left(x, x^{\prime}, A_{i}, b_{i}\right)\right]^{+}-\left[A_{i} x+b_{i}\right]^{+} \\
& \leqslant\left[\left[A_{i} x^{\prime}+b_{i}\right]^{+}-g_{i}\left(x, x^{\prime}, A_{i}, b_{i}\right)\right]^{+}-\left[A_{i} x+b_{i}\right]^{+}=0 .
\end{aligned}
$$

Во втором случае $N\left(x^{\prime}\right) \backslash N(x)=\varnothing$. Тогда $N(x) \neq \varnothing$. Зафиксировав произвольный индекс $q \in N(x)$, элементы пары $\left(A^{\prime}, b^{\prime}\right)$ определим по правилам

$$
\begin{aligned}
a_{i j}^{\prime} & = \begin{cases}\xi_{i}, & \text { если } A_{i} x+b_{i} \geqslant 0, j=q, \\
0 & \text { в остальных случаях, }\end{cases} \\
b_{i}^{\prime} & = \begin{cases}-\xi_{i}, & \text { если } A_{i} x+b_{i}<0, \\
0 & \text { в остальных случаях. }\end{cases}
\end{aligned}
$$


Отсюда, учитывая неравенства (9), для любого индекса $i \in N_{m}$ находим, что если $A_{i} x+$ $b_{i} \geqslant 0$, то

$$
\begin{aligned}
g_{i}\left(x, x^{\prime}, A_{i}+A_{i}^{\prime}, b_{i}+b_{i}^{\prime}\right) & =\left[A_{i} x^{\prime}+b_{i}\right]^{+}-\left(A_{i} x+b_{i}\right)-\xi_{i} \\
& <\left[A_{i} x^{\prime}+b_{i}\right]^{+}-\left(A_{i} x+b_{i}\right)-g_{i}^{+}\left(x, x^{\prime}, A_{i}, b_{i}\right)=0,
\end{aligned}
$$

а если $A_{i} x+b_{i}<0$, то

$$
\begin{aligned}
g_{i}\left(x, x^{\prime}, A_{i}+A_{i}^{\prime}, b_{i}+b_{i}^{\prime}\right) & =\left[A_{i} x^{\prime}+b_{i}-\xi_{i}\right]^{+}-\left[A_{i} x+b_{i}-\xi_{i}\right]^{+} \\
& =\left[A_{i} x^{\prime}+b_{i}-\xi_{i}\right]^{+} \leqslant\left[A_{i} x^{\prime}+b_{i}-g_{i}^{+}\left(x, x^{\prime}, A_{i}, b_{i}\right)\right]^{+} \\
& \leqslant\left[\left[A_{i} x^{\prime}+b_{i}\right]^{+}-g_{i}^{+}\left(x, x^{\prime}, A_{i}, b_{i}\right)\right]^{+} \\
& \leqslant\left[\left[A_{i} x^{\prime}+b_{i}\right]^{+}-g_{i}\left(x, x^{\prime}, A_{i}, b_{i}\right)\right]^{+}=\left[-\left[A_{i} x+b_{i}\right]^{+}\right]^{+}=0 .
\end{aligned}
$$

Очевидно, что в обоих случаях вектор $x \notin S^{m}\left(A+A^{\prime}, b+b^{\prime}\right)$ и $\left\|A^{\prime}\right\|+\left\|b^{\prime}\right\|=\xi<\varepsilon$, то есть $\left(A^{\prime}, b^{\prime}\right) \in \Omega(\varepsilon)$.

Лемма 3 доказана.

Теорема 1. Для радиуса устойчивости любого строго эффективного решения $x \in S^{m}(A, b), m \geqslant 1$, справедлива формула

$$
\rho^{m}(x, A, b)=\min _{x^{\prime} \in X \backslash\{x\}} \alpha\left(x, x^{\prime}, A, b\right) .
$$

Доказательство. Поскольку $x \in S^{m}(A, b)$, для любого решения $x^{\prime} \neq x$ существует такой индекс $i \in N_{m}$, что $g_{i}\left(x, x^{\prime}, A_{i}, b_{i}\right)<0$. Поэтому правая часть формулы (10) является положительным числом, которое будем для краткости обозначать через $\varphi$.

Сначала докажем неравенство $\rho^{m}(x, A, b) \geqslant \varphi$. В соответствии с определением числа $\varphi$, для всякого решения $x^{\prime} \neq x$

$$
0<\varphi \leqslant \alpha\left(x, x^{\prime}, A, b\right) .
$$

Поэтому в силу леммы 2 для любого решения $x^{\prime} \neq x$ справедлива формула (8). Отсюда заключаем, что для любой пары $\left(A^{\prime}, b^{\prime}\right) \in \Omega(\varphi)$ решение $x \in S^{m}\left(A+A^{\prime}, b+b^{\prime}\right)$, то есть $\rho^{m}(x, A, b) \geqslant \varphi$.

Далее покажем, что $\rho^{m}(x, A, b) \leqslant \varphi$. Пусть $\varepsilon>\varphi$ и решение $x^{\prime} \neq x$ таково, что

$$
\alpha\left(x, x^{\prime}, A, b\right)=\varphi \text {. }
$$

Тогда существуют такие положительные числа $\xi_{i}>g_{i}^{+}\left(x, x^{\prime}, A_{i}, b_{i}\right), i \in N_{m}$, что

$$
\varphi<\xi=\sum_{i \in N_{m}} \xi_{i}<\varepsilon
$$

Поэтому по лемме 3 найдется такая пара $\left(A^{\prime}, b^{\prime}\right) \in \Omega(\varepsilon)$, что $x \notin S^{m}\left(A+A^{\prime}, b+b^{\prime}\right)$.

Резюмируя, убеждаемся в справедливости неравенства $\rho^{m}(x, A, b)<\varepsilon$ для всякого числа $\varepsilon>\varphi$. Поэтому $\rho^{m}(x, A, b) \leqslant \varphi$.

Теорема доказана.

Следствие 1. Любое строго эффективное решение $x \in S^{m}(A, b)$ устойчиво, иньми словами, $\rho^{m}(x, A, b)>0$.

Замечание 1. Легко видеть, что число $\varphi$ (правая часть формулы (10)) является нижней оценкой радиуса устойчивости эффективного (оптимального по Парето) решения задачи (1), при этом возможно равенство $\varphi=0$. 


\section{Список литературы}

1. Сергиенко И. В., Козерацкая Л. Н., Лебедева Т. Т., Исследование устойчивости и параметрический анализ дискретных оптимизачионных задач. Наукова думка, Киев, 1995.

2. Sotskov Yu. N., Leontev V. K., Gordeev E. N., Some concepts of stability analysis in combinatorial optimization. Discrete Appl. Math. (1995) 58, 169-190.

3. Greenberg H. J., An annotated bibliography for post-solution analysis in mixed integer programming and combinatorial optimization. In: Advances in Computational and Stochastic Optimization, Logic Programming and Heuristic Search. Kluwer, Boston, 1998, pp. 97-148.

4. Емеличев В. А., Подкопаев Д. П., Устойчивость и регуляризация векторных задач целочисленного линейного программирования. Дискретный анализ и исследование операчий (2001) 8, №1, 47-69.

5. Emelichev V. A., Girlich E., Nikulin Yu. V., Podkopaev D. P., Stability and regularization of vector problems of integer linear programming. Optimization (2002) 51, №4, 645-676.

6. Емеличев В. А., Похилько В. Г., Анализ чувствительности решений векторной задачи минимизации линейных форм на множестве подстановок. Дискретиая математика (1998) 12, №3, $37-48$.

7. Емеличев В. А., Степанишина Ю. В., Многокритериальные комбинаторные линейные задачи: параметризация принципа оптимальности и устойчивость эффективных решений. Дискретиая математика (2001) 13, №4, 43-51.

8. Емеличев В. А., Кричко В. Н., Радиус устойчивости эффективного решения векторной квадратичной задачи булева программирования. Журиал вычисл. матем. и матем. физики (2001) 41, №2, 346-350.

9. Бухтояров С. Е., Емеличев В. А., Степанишина Ю. В., Вопросы устойчивости векторных дискретных задач с параметрическим принципом оптимальности. Кибериетика и системный анализ (2003) №4, 155-166.

10. Емеличев В. А., Кузьмин К. Г. Леонович А. М., Устойчивость в векторных комбинаторных задачах оптимизации. Автоматика и телемеханика (2004), №2, 79-92.

11. Еремин И. И., Мазуров В. Д., Астафьев Н. Н., Несобствепиые задачи липейиого и выпуклого программирования. Наука, Москва, 1983.

12. Бердникова Е. А., Еремин И. И., Попов Л. Д., Распределенные фейеровские процессы для систем линейных неравенств и задач линейного программирования. Автоматика и телемехапика (2004), №2, 16-32.

13. Smale S., Global analysis and economics, V. Pareto theory with constraints. J. Math. Economics (1974), №1, 213-221.

14. Подиновский В. В., Ногин В. Д., Парето-оптимальиые решения миогокритериальиых задач. Наука, Москва, 1982.

15. Гордеев Э. Н., Исследование устойчивости задачи о кратчайшем остове в метрике $l_{1}$. Журпал вычисл. матем. и матем. физики (1999) 39, №5, 770-778.

16. Гордеев Э. Н., Исследование устойчивости оптимизационных задач на матроидах в метрике $l_{1}$. Кибернетика и системпый апализ (2001) №2, 132-144. 